\begin{tabular}{|c|c|}
\hline Title: & $\begin{array}{l}\text { Increasing the operating range of permanent magnet synchronous motors by switching } \\
\text { the winding configurations }\end{array}$ \\
\hline Authors: & Miriam Boxriker, Patrick Winzer, Johannes Kolb, Martin Doppelbauer \\
\hline Institute: & $\begin{array}{l}\text { Karlsruhe Institute of Technology (KIT) } \\
\text { Elektrotechnisches Institut (ETI) } \\
\text { Hybrid Electric Vehicles (HEV) }\end{array}$ \\
\hline Type: & Conference Proceedings \\
\hline Published at: & $\begin{array}{l}2016 \text { IEEE 2nd Annual Southern Power Electronics Conference (SPEC), Auckland, } 2016 \\
\text { Publisher: IEEE, Piscataway (NJ) } \\
\text { Year: } 2016 \\
\text { ISBN: } 978-1-5090-1546-7 \\
\text { Pages: } 1-6\end{array}$ \\
\hline Hyperlinks: & DOI: https://doi.org/10.1109/SPEC.2016.7846062 \\
\hline
\end{tabular}

(C) 2016 IEEE. Personal use of this material is permitted. Permission from IEEE must be obtained for all other uses, in any current or future media, including reprinting/republishing this material for advertising or promotional purposes, creating new collective works, for resale or redistribution to servers or lists, or reuse of any copyrighted component of this work in other works. 


\title{
Increasing the Operating Range of Permanent Magnet Synchronous Motors by Switching the Winding Configurations
}

\author{
Miriam Boxriker, Patrick Winzer, Johannes Kolb* and Martin Doppelbauer \\ Elektrotechnisches Institut (ETI) - Hybrid Electric Vehicles (HEV) \\ Karlsruhe Institute of Technology \\ Kaiserstr. 12, 76131 Karlsruhe, Germany \\ Email: miriam.boxriker@kit.edu \\ ${ }^{*}$ Schaeffler Technologies AG \& Co. KG - SHARE at KIT
}

\begin{abstract}
This paper presents a holistic approach to determine the effect of online reconfiguration of the motor winding of a synchronous permanent magnet motor resulting in switching the number of turns. The analysis is based on a parameter plane, which predicts the behavior of synchronous machine designs by using only two normalized parameters, the permanent magnet flux linkage and the ratio of the quadrature axis inductance $L_{\mathrm{q}}$ to the direct axis inductance $L_{\mathrm{d}}$. This enables a fast and reliable evaluation of all alternate winding configurations under given constraints. By generating a Pareto front, superior switching configurations can be identified. An exemplary configurable winding machine - implemented in a finite element analysis (FEA) - shows a significant improvement with respect to maximum torque (increase by $20.3 \%$ ) and usable torque-speed operating range (increase by $5.5 \%$ ) compared to the best fixed-winding configuration. The contribution demonstrates that this approach is not only applicable for permanent magnet synchronous motors but also for synchronous reluctance, electrically excited synchronous and induction motors.
\end{abstract}

\section{INTRODUCTION}

Changing the winding configuration has a long history in the section of grid-connected motors. For example, they have served as a starting configuration for induction motors (IM) using the star-delta-reconfiguration [1]. Moreover, they were the means of choice for a simple change of the operation point without power electronics by switching the number of pole-pairs [1]. Recently, since the efforts of increasing the utilization of electric motors grow especially in the field of traction drives, the switching of the winding configurations is again in the focus of research. By changing the arrangement of the motor coils, the stator number of turns in series per phase $w[1]$ is altered, while the input power - current and voltage per phase - remain constant, leading to a different torque-speed-characteristic of the motor. The described setup is depicted in Fig. 1. A power converter supplies the motor, providing a maximum voltage and a maximum power per phase. Furthermore, there is a unit enabling the switching of the coil configurations which is independent of the main power electronics. This is exemplarily shown for a star-deltaconfiguration resulting in $w_{\mathrm{Wye}}=w$ and $w_{\Delta}=w / \sqrt{3}$. For example, the series configuration of the stator coils of the phases is changed during operation to a parallel configuration resulting in halving $w$ as shown in Fig. 2. This eventuates in two different torque-speed-characteristics and by choosing the design in a sensible way - in a widening of the operating range.

Permanent magnet synchronous motors (PMSM) offer high efficiency, high torque and power density on the one hand, but on the other hand they lack the performance capabilities of IMs or electrically excited synchronous motors (EESM) in the field weakening area. Therefore, PMSMs are the preferred machine type to examine the effects of a switched winding configuration [2]. A variable coil configuration holds the opportunity to merge the mentioned advantages of PMSMs with an improved field weakening capability. [2] presents a surface PMSM, capable of changing the coil configuration from star to delta including a series or a parallel connection of the phase coils, which could extend its operating area to higher speed values. While [3] also concentrates on the motor, [4], [5] introduce possible winding switching techniques.

However, although those works show an increase in the operating capability of the examined systems, they only analyzed one specific solution. [6], [7] move a step further by introducing a factor for the change of $w$ due to the coil configuration change. By deriving an extension of the parameter plane, which is initially presented in [8], they achieve an analytical prediction of the torque-speed-characteristic. The

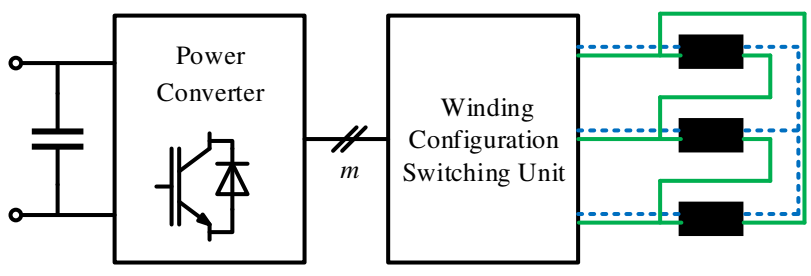

Figure 1. The setup for operating the motor with a variable coil configuration, e.g. $w_{\Delta}=w / \sqrt{3}$ configuration and $w_{\mathrm{Wye}}=w$ configuration (dashed). 

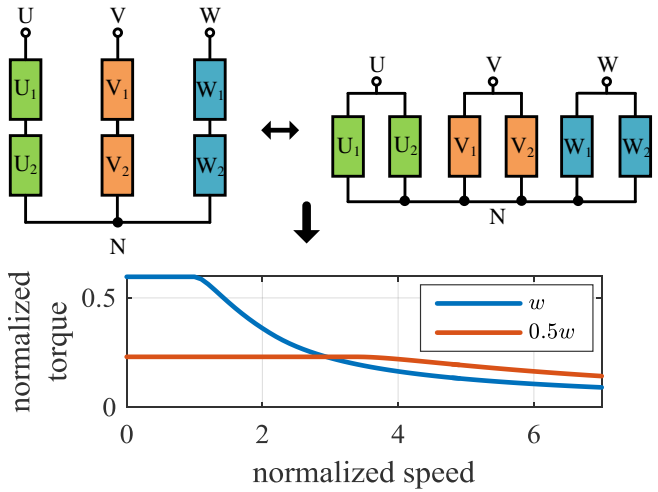

Figure 2. Changing $w$ by 2 , respectively $1 / 2$ due to a series - parallel change of the coils $(\mathrm{U} 1, \mathrm{U} 2, \ldots)$ of the three phases $\mathrm{U}, \mathrm{V}, \mathrm{W}$ leading to different torquespeed-characteristics

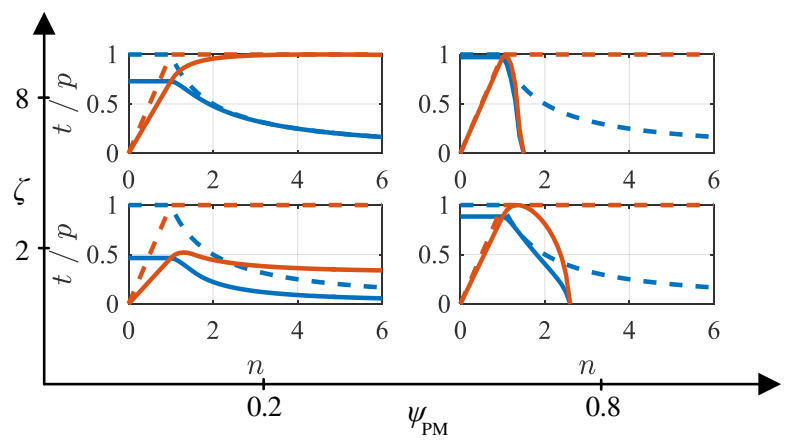

Figure 3. Normalized power $(p)$ - torque $(t)$ - characteristics in the parameter plane dependent on $\psi_{\mathrm{PM}}$ and $\zeta$ with the ideal characteristics in dashed lines

parameter plane enables the motor designer to describe the behavior of synchronous machines by only two parameters: the permanent magnet flux linkage $\psi_{\mathrm{PM}}$ and the saliency ratio of the motor inductances $\zeta=L_{\mathrm{q}} / L_{\mathrm{d}}$ by normalizing the machine parameters. The extension includes the changing of $w$ into the plane and calculates its effects concerning the torque-speed-characteristic. In Fig. 3 the different torquespeed-characteristics dependent on $\psi_{\mathrm{PM}}$ and $\zeta$ are shown.

The purpose of this contribution is therefore to offer benchmark criteria to quickly evaluate the results of deliberate winding configuration changes and to show the applicability not only on PMSM but also on other motor types. It also describes the derivation of the ratio factor $k$ for ratio of $w$ of the different winding configurations and considers also machines with a phase number greater than three. First, the parameter plane and its expansion are explained in the following Section, including also two benchmark criteria evaluating the benefit of the coil configuration switch. Subsequently, the ratio of the winding configuration is explained. Then, the results are presented by using a Pareto optimization and conclusions are made concerning the potential and benefit of switching the winding configuration of a machine. Moreover, the applicability on other machine types is shown. Finally, the approach is verified by a Finite-Element Analysis (FEA).

\section{The Parameter Plane}

For the calculation of the parameter plane, the following has to be assumed: All losses are set to zero, while the iron and permanent magnets are considered linear [9]. Moreover, the higher harmonics are set to zero and all physical values are sinusoidal respectively. The well-known motor equations for describing synchronous motors [8] have to be normalized in that way that the maximum current $i_{\max }$, the maximum voltage $v_{\max }$, the maximum ideal torque $t$ and power are set to one in order to be able to compare different motor designs. That means that the maximum base speed is also normalized to one and the field weakening area starts at speed $\omega=1$. That leads to these equations:

$$
\begin{gathered}
v_{\mathrm{d}}=-\omega \zeta l_{\mathrm{d}} i_{\mathrm{q}} \\
v_{\mathrm{q}}=\omega l_{\mathrm{d}} i_{\mathrm{d}}+\omega \psi_{\mathrm{PM}} \\
t=\psi_{\mathrm{PM}} i_{\mathrm{q}}-(\zeta-1) l_{\mathrm{d}} i_{\mathrm{d}} i_{\mathrm{q}}
\end{gathered}
$$

where $l_{\mathrm{d}}$ is the normalized direct inductance, $v_{\mathrm{d}}$ and $v_{\mathrm{q}}$ are the normalized voltages in $\mathrm{d}$ - and $\mathrm{q}$-axis and $i_{\mathrm{d}}$ and $i_{\mathrm{q}}$ are the normalized currents in the specified axes. The control is determined by using the maximum torque per ampere (MTPA) strategy [8], since the following constraints are active:

$$
\begin{gathered}
\sqrt{v_{\mathrm{d}}^{2}+v_{\mathrm{q}}^{2}} \leq v_{\max }=1 \\
\sqrt{i_{\mathrm{d}}^{2}+i_{\mathrm{q}}^{2}} \leq i_{\max }=1
\end{gathered}
$$

[8] showed that the motor equations consist of only two independent parameters: $\psi_{\mathrm{PM}}$ and $\zeta$, since $l_{\mathrm{d}}$ is dependent of $\psi_{\mathrm{PM}}$ and $\zeta$ and is therefore calculated. $\psi_{\mathrm{PM}}$ and $\zeta$ consequently span a parameter plane, where the motor characteristics are presented (Fig. 2 and Fig. 3).

\section{A. The Extension of the Parameter Plane}

First, the motor equations have to be adapted regarding the effects of switching the winding configuration. This is similarly done by [6]. The motor equations must not be normalized each separately but to a base turn number $w_{\mathrm{b}}$. This ensures that several designs with various $w$ are depicted with the right relation with respect to torque, $\omega_{0}$ and the field weakening behavior. The dependence of $\psi_{\mathrm{PM}}$ and the absolute inductances $L_{(\mathrm{d}, \mathrm{q})}$ on $w$ can be written as $\psi_{\mathrm{PM}} \propto w$ and $L_{(\mathrm{d}, \mathrm{q})} \propto w^{2}$ [1]. Thus, the change of $w$ is implemented as a ratio factor $k$ with $0<k \leq 1$, where the greatest occurring $w$ corresponds to $w_{\mathrm{b}}$ and therefore to $k=1$. Consequently, the equations (1) to (3) change to:

$$
\begin{gathered}
v_{\mathrm{d}}=-\omega \zeta k^{2} l_{\mathrm{d}} i_{\mathrm{q}} \\
v_{\mathrm{q}}=\omega k^{2} l_{\mathrm{d}} i_{\mathrm{d}}+\omega k \psi_{\mathrm{PM}} \\
t=k \psi_{\mathrm{PM}} i_{\mathrm{q}}-(\zeta-1) k^{2} l_{\mathrm{d}} i_{\mathrm{d}} i_{\mathrm{q}}
\end{gathered}
$$


Every configuration can be written in this form, as only the ratio from the different existing $w$ to each other is important. Hence, $k$ has only discrete and distinctive values, since the change of winding results in a different arrangement of the coils of a phase. The value of $l_{d}$ does not change, as it is carried out for $k=1$ by using the MTPA-strategy. $v_{\max }$ and $i_{\max }$ as well as the normalizing factors remain the same for different $k$, whereas $i_{(\mathrm{q}, \mathrm{k})}$ and $i_{(\mathrm{d}, \mathrm{k})}$ are dependent of $k$. Therefore, they and the control have to be calculated for each occurring $k$ separately. Then the maximum base speed $\omega_{0}(k)$ is computed for each $k$ by:

$$
\omega_{0}(k)=\frac{1}{\sqrt{\left(\zeta k^{2} l_{\mathrm{d}} i_{\mathrm{q}}\right)^{2}+\left(k^{2} l_{\mathrm{d}} i_{\mathrm{d}}+k \psi_{\mathrm{PM}}\right)^{2}}}
$$

The maximum torque curves for $k$ are also obtained in the field weakening area with the MTPA- and the MTPV- (Maximum Torque per Voltage) strategy respectively [8].

\section{B. Optimization Criteria}

Since the aim of this contribution is to find an optimum design for specific constraints with regard to the motor parameters, the effect of switching the winding configuration has to be made matchable. As explained before, the configuration change results in different torque-speed-characteristics which evaluating criteria have to consider. In this paper, two possible criteria are introduced which fulfill the demands of achieving a comparable benchmark value. The first criterion deals with the size of the operating area of the motor spanned by the different torque-speed-curves. The other criterion judges the maximal divergence from the ideal torque-speed-curve. Both criteria are explained in the following.

1) Criterion of Operating Range: This criterion estimates the achievable operating area of a motor design with a specific winding configuration switch $\vec{k} . \vec{k}$ is the vector of the ratio factor $k$, holding all possible values of $k$ for a certain design with a variable winding. That allows an assessment of the utilization of a motor design by comparing it to the ideal operating area. The operating area $A_{\mathrm{P}}$ is calculated by

$$
A_{\mathrm{P}}=\int_{0}^{\omega_{\mathrm{end}}} t_{\max }(\omega, \vec{k}) \mathrm{d} \omega
$$

with the maximum speed $\omega_{\text {end }}$ for a spreading $s=\frac{\omega_{\text {end }}}{\omega_{0}}$, as the maximum attainable speed $\omega_{\text {end }}$ and the maximum base speed are mostly predefined design criteria. $t_{\max }$ is the maximum torque-speed-curve resulting from the particular maximum torque $t(\omega, k)$ at each speed of the different winding configurations with various $w$. An example is given in Fig. 4. The integral of $t_{\max }(\omega, \vec{k}), A_{\mathrm{P}}(\vec{k})$, is a measure of the size of the operating area which is achieved by the change of $w$. To determine the effect and to be able to compare the results, $A_{\mathrm{P}}(\vec{k})$ is normalized to $A_{\mathrm{P}, \text { ideal }}$, the $A_{\mathrm{P}}$ of the ideal motor torque-speed-characteristic, which is $t_{\text {ideal }}(\omega=0 \ldots 1)=1$ and

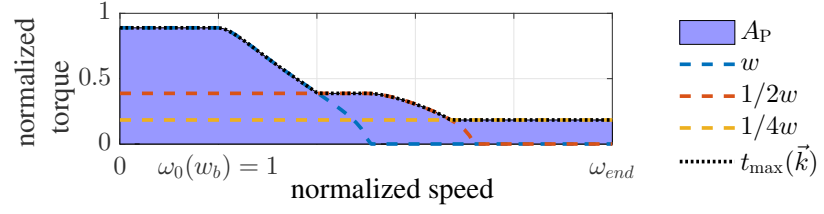

Figure 4. Picture of operating range criterion $A_{\mathrm{P}}$ and the maximum torque $t_{\text {max }}$ with winding configuration change of $\vec{k}=\left\{1, \frac{1}{2}, \frac{1}{4}\right\}$

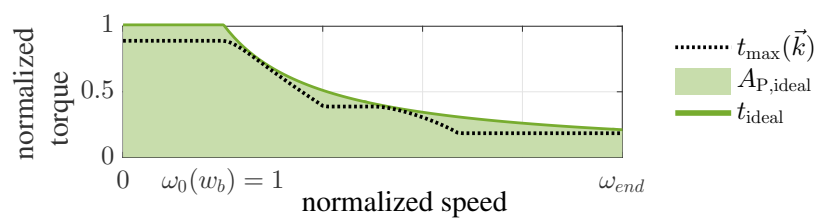

Figure 5. Picture of ideal operating range $A_{\mathrm{P} \text {,ideal }}$ and the ideal torque $t_{\text {ideal }}$ compared to $t_{\max }$ of Fig. 4

$t_{\text {ideal }}(\omega>1)=1 / \omega$, as it is depicted in Fig. 5. This yields the normalized integral $A_{\mathrm{Pn}}$ :

$$
A_{\text {Pn }}=\frac{\int_{0}^{\omega_{\text {end }}} t_{\max }(\omega, \vec{k}) \mathrm{d} \omega}{\int_{0}^{\omega_{\text {end }}} t_{\text {ideal }}(\omega) \mathrm{d} \omega}
$$

$A_{\mathrm{Pn}}(\vec{k}=1)$ represents the maximum operating area which can be attained without changing the winding configuration.

2) Criterion of Ideal Torque Divergence: Another way to judge the outcome of the calculation, is the maximal divergence from a requested torque-speed-curve, whereas this curve is set to the ideal torque-speed-curve. An example is given in Fig. 6. This criterion is similar to the evaluation of the continuous power speed range (CPSR) [8], as it judges the continuity of the field weakening area as well. Additionally, it takes the value of the base torque into account leading to

$$
V_{\text {Pn }}=\max \left(t_{\text {ideal }}(\omega)-t_{\max }(\omega, \vec{k})\right)
$$

The reason to use this criterion is to fit the machine design best to the ideal torque-speed-curve. If the motor design curve does not fit the requirements - assumed that the input ratings stay fixed - the torque can only be enhanced by enlarging the volume of the rotor, which is proportional to the output torque. Therefore, $V_{\mathrm{Pn}}$ is a measure of the needed rotor size and moreover, a first approximation of the size of the whole machine. This can be seen as a mean of dealing with restricted space requirements.

\section{Ratios of Switched Winding Configurations}

For a specific design, there are only several possibilities in arranging the coils, which is examined in this section. Their number and arrangements find expression in various numbers of turns in series per phase $w$ and therefore in the vector $\vec{k}$. It has to be considered, that also a miscellaneous input power or a different proportion of voltage and current with a fixed winding configuration can be likewise converted into a change of $w$. Determining the vector is essential for calculating the presented criteria. $\vec{k}$ is dependent on the phase number 


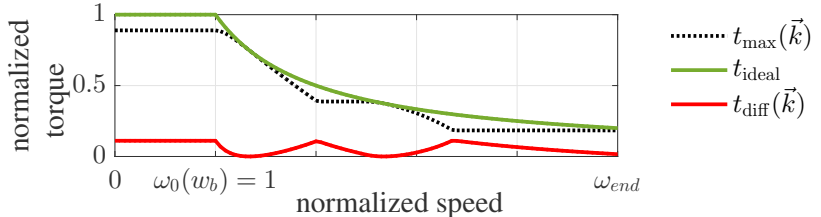

Figure 6. Picture of $V_{\mathrm{pn}}$ criterion for $\vec{k}=\left\{1, \frac{1}{2}, \frac{1}{4}\right\}$, which is the maximum of $t_{\text {diff }}(\omega, \vec{k})$

and the number of coils per phase. It is presumed that the input power does not change during operation, that the phase current and phase voltage stay constant and that only the coil current and coil voltages are changing due to the switched coil configurations. As stated in [10], multiphase machines with a phase number $>3$ have several advantages compared to three-phase motors, e.g. higher torque, better utilization of DC voltage, lower torque ripple and lower double-linkage leakage. Therefore, not only three-phase-machines are taken into account but also higher phase numbers. The two main effects are described in the following:

\section{A. Influence of Coil Number per Phase}

The number of coils per phase $n_{\mathrm{c}}$ determines which series and parallel configuration are conceivable. In the following, it is assumed that the back-EMFs in the single coils of one phase are the same at each point of time due to the design. This avoids additional balancing currents. If there are two coils per phase, there are two configurations executable: a series and a parallel configuration. If there are three coils per phase, an additional configuration emerges, which is a combination of a series and a parallel configuration. Which coils are in the series or parallel configuration, does not influence the result of $\vec{k}$. Tab. I lists the resulting arrangements and $\vec{k}$. Hence, $n$ coils per phase lead to $n$ different configurations with variable $k$. However, some configurations yield unbalanced load of the coils, e.g. the second configuration at $n_{\mathrm{c}}=3$ or $n_{\mathrm{c}}=4$. Therefore, those configurations are rather an academic than a real world approach. Nevertheless, those $\vec{k}$ can always be realized with a higher number of coils without an unbalanced stress.

\section{B. Influence of Phase Number}

Furthermore, the way of connecting the phases between one another effects the amount of voltage and current over a phase at a constant DC link voltage (see Fig. 1). At a constant input power, the proportion of available current and voltage per phase is therefore alternated which directly affects the motor operation and the torque-speed-curve. Consequently, this can be expressed by a change of $w$. There is always the possibility to connect all phases in a common point (Wye-configuration), which value can be set to one at first. The other values of vector $\vec{k}$ can be calculated by determining the inverse of the interlinking factor $r_{\mathrm{VF}}=\sqrt{2(1-\cos \gamma)}$, whereas $\gamma$ is the angle between the two single phases. They represent possible polygon configurations.
Table I

THE RELATION OF COIL NUMBER PER PHASE $n_{\mathrm{C}}$ AND THE CONFIGURATIONS $\vec{k}$

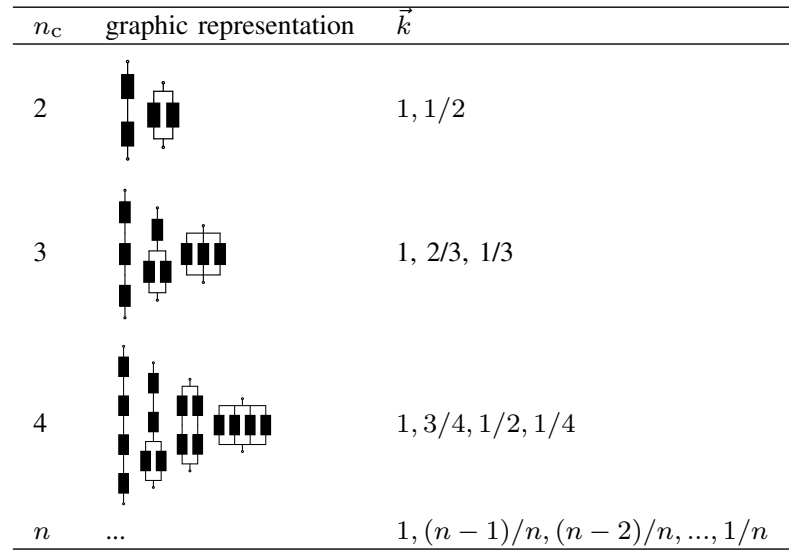

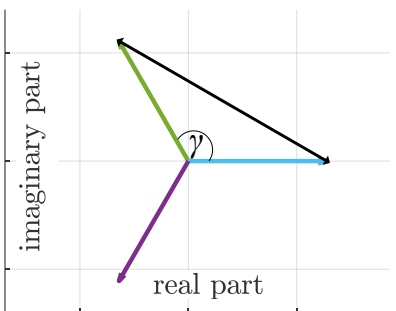

one interlinking factor

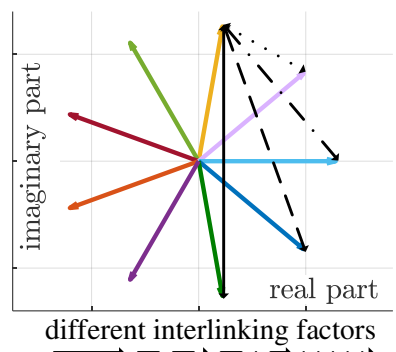

different interlinking factors

Figure 7. Three-phase voltages lead- Figure $8 . m$-phase voltages (here ing to only one interlinking factor. $\quad m=9$ ) leading to different interlinking factors

- $m=3$ : If the phase number is three, there is only one interlinking factor $r_{\mathrm{VF}}=\sqrt{3}$ as can be seen in Fig. 7 . Thus, there are two configurations in which the phases can be connected to each other: the Wye- $\Delta$-configuration resulting in $\vec{k}=\{1,1 / \sqrt{3}\}$.

- $m>3$ : Fig. 8 exemplarily shows $m=9$ phases. Here, there are four different interlinking factors resulting in four different polygon configurations and one star-point configuration. In contrast to a three-phase-machine, a multiphase machine offers more opportunities in combining the phases.

Both influences, the number of coils per phase and the phase number, add up in a resulting vector $\vec{k}$ for the given constraints. For example, a nine phase machine with two coils per phase holds a maximum of ten different configurations with $\vec{k}=$ $\{1,0.684,0.532,0.5,0.395,0.347,0.342,0.266,0.197,0.174\}$

\section{RESUlts OF PARETO OPTIMIZATION}

The previous Section allows calculating the effect of a switched winding configuration by determining the proposed benchmark criteria. At least, this is a problem with four degrees of freedom, yielding the following parameters:

- $\psi_{\mathrm{PM}}$ and $\zeta$, the two parameter spanning a parameter plane 

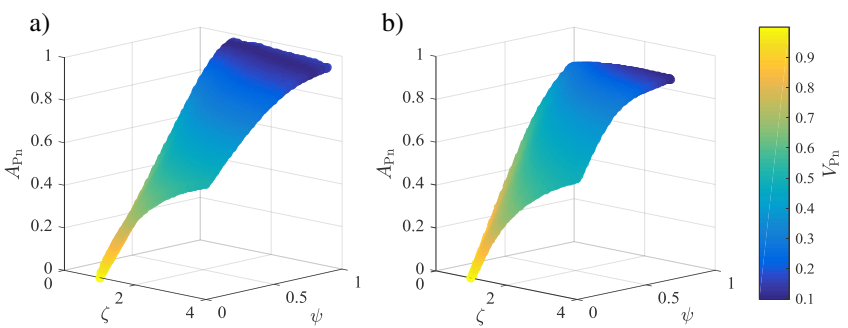

c)

d)
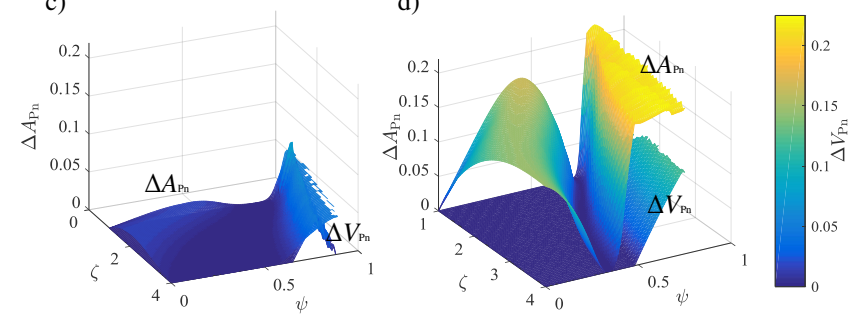

Figure 9. Results of Pareto optimality for $\overrightarrow{\mathbf{k}}$ consisting of all switching configurations for $m=9$ and two coils per phase a) with $s=2$ b) with $s=9$ and the difference between a variable coil configuration and a fixed configuration in the $A_{\mathrm{Pn}}, \Delta A_{\mathrm{Pn}}$, and $V_{\mathrm{Pn}}$ criterion, $\left.\Delta V_{\mathrm{Pn}}, \mathrm{c}\right)$ for $s=2 \mathrm{~d}$ ) for $s=9$

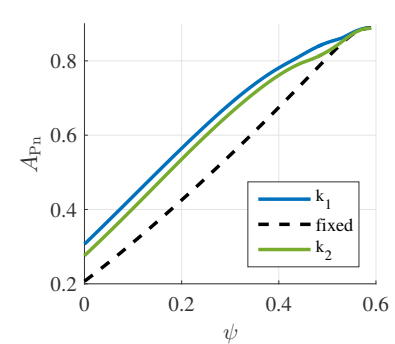

Figure 10. Pareto front with $\vec{k}_{1}=$ $\{1,0.778,0.5077,1 / 2,0.389,0.2539\}$ and $\vec{k}_{2}=\{1,1 / 2\}$ of $A_{\mathrm{Pn}}$ with $s=9$ and $\zeta=2$

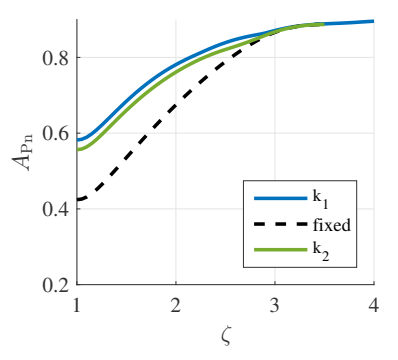

Figure 11. Pareto front with $\vec{k}_{1}=$ $\{1,0.778,0.5077,1 / 2,0.389,0.2539\}$ and $\vec{k}_{2}=\{1,1 / 2\}$ of $A_{\mathrm{Pn}}$ with $s=9$ and $\psi_{\mathrm{PM}}=0.4$
- $\overrightarrow{\mathbf{k}}$, a $n$-tuple of vectors $\vec{k}$, holding all allowed winding configurations for designs that should be examined

- $s=\frac{\omega_{\text {end }}}{\omega_{0}}$, the spreading which influences the constraints of the criteria

- the results and their dependencies are $A_{\mathrm{Pn}}=$ $f\left(\psi_{\mathrm{PM}}, \zeta, \overrightarrow{\mathbf{k}}, s\right)$ or $V_{\mathrm{Pn}}=f\left(\psi_{\mathrm{PM}}, \zeta, \overrightarrow{\mathbf{k}}, s\right)$

In order to be able to evaluate the results without losing valuable data, the degrees of freedom of the problem have to be reduced. This is done by finding the Pareto optimality and displaying the Pareto front. This front shows only results of a parameter set, which is not dominated by the result of another parameter set. It is sensible to choose the number of winding configuration, $\psi_{\mathrm{PM}}, \zeta$ and the criteria as objectives. That means that the criteria can be reached by the lowest possible amount of switching configurations, the lowest $\psi_{\mathrm{PM}}$ and $\zeta$. A reduced $\psi_{\mathrm{PM}}$ coincides with less magnet material or less stator windings reducing the needed stator slot size. A decreased saliency implies a simpler geometric structure and less influence of saturation effects.

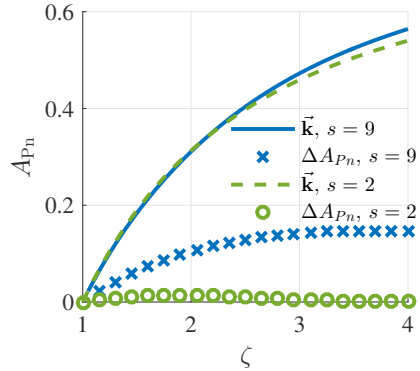

Figure 12. Pareto Front of SynRM and IM for $\overrightarrow{\mathbf{k}}$ consisting of all switching configurations for $m=3$ and four coils per phase for $s=2$ and $s=9$

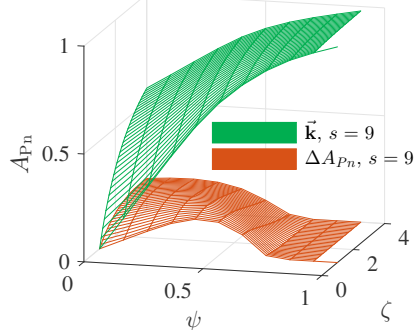

Figure 13. Pareto Front of EESM for $\overrightarrow{\mathbf{k}}$ consisting of all switching configurations for $m=3$ and $n_{\mathrm{c}}=4$ with $s=9$ and $\Delta A_{P n}$
Two Pareto fronts are deployed in Fig. 9a) and b) with the mentioned objectives to explain the dependencies of the different parameter on the criteria. Although an unconstrained number of tuples of $\vec{k}, \overrightarrow{\mathbf{k}}$, could be calculated and shown, these Pareto fronts are limited to all coil configurations given by $m=9$ and two coils per phase. While the ratio between $\omega_{\text {end }}$ and $\omega_{0}, s$, is two in Fig. 9a), it is increased to nine in Fig. 9b). Fig. 9c) and Fig. 9d) depict the benefit of the variable configuration at $s=2$ and $s=9$ compared to a fixed winding configuration regarding the criteria $A_{\mathrm{Pn}}, \Delta A_{\mathrm{Pn}}$, and $V_{\mathrm{Pn}}, \Delta V_{\mathrm{Pn}}$. If the needed maximum speed is not substantially bigger than the maximum base speed, e.g. $s=2$, the effect of changing $w$ is small for $\psi_{\mathrm{PM}}<0.8 . \Delta A_{\mathrm{Pn}}$ only rises for $\psi_{\mathrm{PM}}$ nearly one. The higher $s$, the bigger the increase in $A_{\mathrm{Pn}}$ with variable $w$ for lower $\psi_{\mathrm{PM}}$ and the higher the reachable profit of more configuration changes.

Fig. 10 and Fig. 11 look at the results from a different angle to show effects on a more detailed level: Both yield the results of $A_{\mathrm{Pn}}$ for two different configurations $\vec{k}_{1}$ and $\vec{k}_{2}$ with the comparison of a fixed winding configuration, whereby $\vec{k}_{2}$ consists of only one configuration change and $\vec{k}_{1}$ of five changes. While only $\psi_{\mathrm{PM}}$ is variable and $\zeta$ is two in Fig. 10, $\psi_{\mathrm{PM}}$ is constant $\left(\psi_{\mathrm{PM}}=0.4\right)$ in Fig. 11 . As can be seen, the saliency or the flux linkage can be reduced by a variable coil configuration at the same flux linkage or the same saliency respectively without decreasing $A_{\mathrm{Pn}}$.

\section{A. Applicability to Other Machine Types}

Other machine types than PMSM can also be considered with this approach. These are the synchronous reluctance motor (SynRM), the induction motor (IM) and the electrically excited synchronous motor (EESM).

- The SynRM is automatically included in the PM approach, as they emerge from setting $\psi_{\mathrm{PM}}$ to zero.

- It was shown by [11], that with approximations IMs can be also described in the parameter plane, coinciding with the same parameter set as SynRMs.

- EESMs with $l_{\mathrm{d}}>l_{\mathrm{q}}$ and a variable $\psi$ are also characterizable in the parameter plane [12].

Fig. 12 displays the results of the Pareto Optimization for SynRM and IM, whereby the configurations $\overrightarrow{\mathbf{k}}$ are chosen 
Table II

MACHINE DATA

\begin{tabular}{ll}
\hline Data & Value \\
\hline rated max. current & $12 \mathrm{~A}$ \\
DC-link voltage & $133 \mathrm{~V}$ \\
base winding number & 18 \\
number of slots & 36 \\
pole pair number & 2 \\
\hline
\end{tabular}

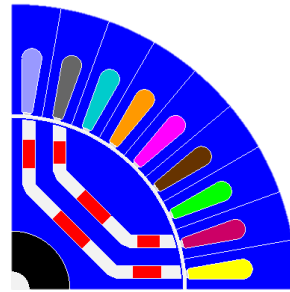

Figure 14. Picture of FEA Design N36m9p2

for three phase motors with four coils per phase. While the enhancement in the operating range by switching the winding configuration stays small for small spreadings, the benefit increases with a rising spreading. That means that a configuration change only pays off with a higher spreading. In contrast to PSM, EESM only profit from a winding configuration change at lower flux linkage values as depicted in Fig. 13. Since an excellent flux weakening capability is inherent in the EESM, there is no way to improve this additionally by a change of $w$. However, at lower flux linkage values the same operating range can be achieved with even less flux linkage if $w$ is variable. That means lower rotor currents and therefore lower losses in the rotor.

\section{Finite Element Analysis}

The previous Section demonstrated the analytically derived benefit of switched coil configurations for increasing the operating range. In order to verify the presented approach and to show the effects of saturation, an exemplary design is built in a finite element (FE) software, Flux2D. A ninephase machine design with the data of Tab. II is chosen displaying also the effect of switched winding configurations on multiphase machines. According to the Pareto front of Fig. 9b), the design with $\psi_{\mathrm{PM}}=0.66$ and $\zeta=4$ is promising, whereby $\vec{k}=\{1,0.684,0.5321,0.5,0.3949,0.3473,0.342$, $0.2661,0.1975,0.1737\}$. Fig. 14 yields the FE-design which fulfills the proposed parameter set at rated current. The normalized results for the mentioned $\vec{k}$ are depicted in Fig. 15a). The torque-speed-characteristics of the analytical approach are shown in Fig. 15b). The mean difference of both maximum torque-speed-characteristics is $1.8 \%$ and the total divergence in the $A_{\mathrm{Pn}}$ criterion is only $1.2 \%$. This is changed for a higher saturation. For example, if the current is five times the rated current for $w_{\mathrm{b}}, \zeta$ and $\psi_{\mathrm{PM}}$ decrease leading to the characteristic in Fig. 15c) with a reduced base torque by $41 \%$ compared to the ideal base torque but an improved field weakening area.

\section{CONCLUSION}

This contribution presents an analytical calculation of the effect of switching the winding configuration, effectively resulting in a change of the number of turns in series per phase $w$. A procedure to determine the optimum number of winding changes and the optimum design for a specific application is
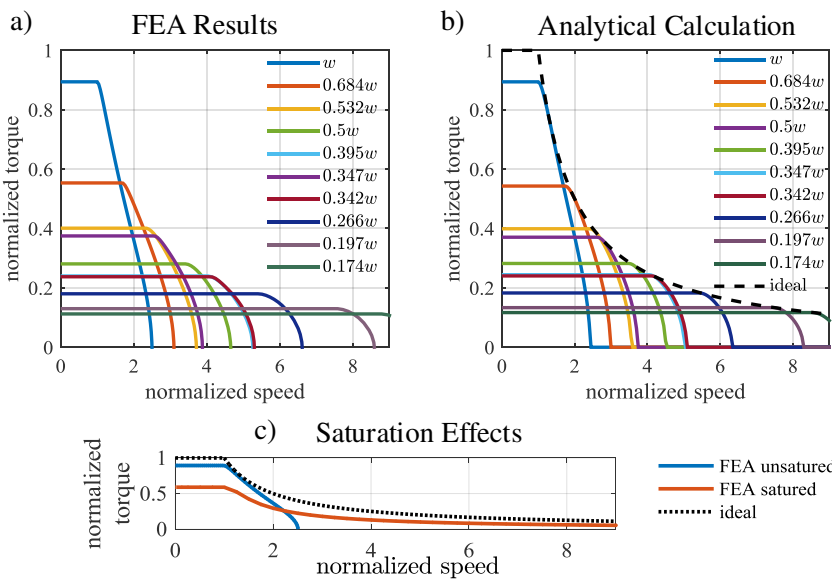

Figure 15. a) Results of the FEA with rated current compared to the analytical calculation in b). c) shows the effects of saturation compared to the ideal torque-speed-characteristic

also submitted. The extended parameter plane can be applied easily and determines quickly all possible gains for a ratio of $\omega_{\text {end }}$ to $\omega_{0}$. An exemplary design of a motor, calculated with a finite element analysis, proofs the applicability of the procedure and also shows the influence of saturation. With this design, the operating range could be improved by $82 \%$.

\section{REFERENCES}

[1] G. Müller, K. Vogt and B. Ponick, Berechnung elektrischer Maschinen, 6th edition, WILEY-VCH Verlag GmbH \& Co. KGaA, Weinheim, 2008

[2] E. Nipp, Permanent Magnet Motor Drives with Switched Stator Windings, Dissertation, Stockholm, 1999.

[3] Y. Miyama, H. Hijikata, Y. Sakai, K. Akatsu, H. Arita and A. Daikoku, Variable characteristics technique on permanent magnet motor for electric vehicles traction system, IEEE International Electric Machines and Drives Conference (IEMDC), 2015, pp. 596-599.

[4] M. M. Swamy, T. Kume, A. Maemura and S. Morimoto, Extended highspeed operation via electronic winding-change method for AC motors, IEEE Transactions on Industry Applications, 2006, pp. 742-752.

[5] B.-I. Kwon, T. A. Lipo and S. Atiq, Experimental verification of winding switching technique to enhance maximum speed operation of surface mounted permanent magnet machines, IET Electric Power Applications, 2016, pp. 294-303.

[6] L. Hao, C. Namuduri, S. Naik and C. Freitas, High Speed Performance of PM Machine with Reconfigurable Winding, IEEE Energy Conversion Congress and Exposition (ECCE), 2015, pp. 1840 - 1848.

[7] L. Hao, C. Namuduri, S. Gopalakrishnan and C. Freitas, Comparison of the Influence of PM Drive System with Voltage Adaptation or Machine Winding Reconfiguration on HEV/EV Applications, IEEE Energy Conversion Congress and Exposition (ECCE), 2015, pp. 6130 - 6138

[8] W. L. Soong, Design and Modelling of Axially-Laminated Interior Permanent Magnet Motor Drives for Field-Weakening Applications, Dissertation, Glasgow, 1993

[9] P. Winzer and M. Doppelbauer, Theoretical Analysis of Synchronous Machines with Displaced Reluctance Axis, International Conference on Electrical Machines, 2014, pp. 641-647.

[10] M. Boxriker, J. Kolb and M. Doppelbauer, Expanding the Operating Range of Permanent Magnet Synchronous Motors by Using the Optimum Number of Phases, 18th European Conference on Power Electronics and Applications, 2016

[11] N. Bianchi and S. Bolognani, Unified Approach to the Analysis and Design of an AC Motor Drive for Flux-Weakening Operations, The 1998 IEEE Industry Applications Conference, 1998, Vol. 1, pp. 95 - 102.

[12] P. Winzer and M. Doppelbauer, Characterisation and Dimensioning of electrically excited synchronous machines as traction drives by means of the normalised parameter plane, Internationaler ETG-Kongress Berlin, 2013, pp. $95-102$ 